\title{
Author Correction: High-power lithium-selenium batteries enabled by atomic cobalt electrocatalyst in hollow carbon cathode
}

Hao Tian (D), Huajun Tian, Shijian Wang, Shuangming Chen, Fan Zhang, Li Song (1D, Hao Liu (D), Jian Liu (D) \& Guoxiu Wang

Correction to: Nature Communications https://doi.org/10.1038/s41467-020-18820-y, published online 6 October 2020.

The original version of this Article omitted Hao Tian and Huajun Tian as equally contributing authors.

This has now been corrected in both the PDF and HTML versions of the Article.

Published online: 28 October 2020

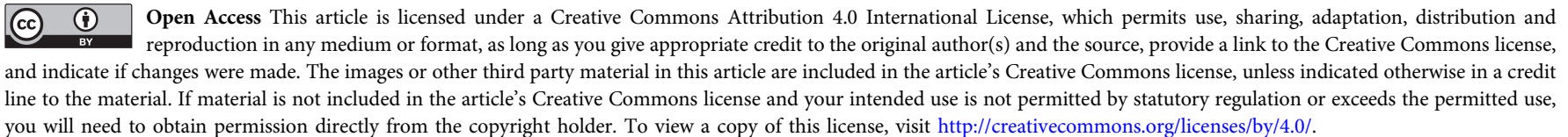
you will need to obtain permission directly from the copyright holder. To view a copy of this license, visit http://creativecommons.org/licenses/by/4.0/.

(c) The Author(s) 2020 